\title{
Metabolomics Investigation of Cutaneous T Cell Lymphoma Based on UHPLC-QTOF/MS
}

\author{
Qing-Yuan Zhou ${ }^{1}$, Yue-Lin Wang ${ }^{1}$ Xia Li ${ }^{1}$, Xiao-Yan Shen ${ }^{2}$, Ke-Jia Li ${ }^{2}$, Jie \\ Zheng $^{2}$, Yun-Qiu Yu ${ }^{1 *}$
}

\begin{abstract}
Objectives: The identification of cutaneous T cell lymphoma (CTCL) biomarkers may serve as a predictor of disease progression and treatment response. The aim of this study was to map potential biomarkers in CTCL plasma. Design and Methods: Plasma metabolic perturbations between CTCL cases and healthy individuals were investigated using metabolomics and ultrahigh performance liquid chromatography-quadrupole time-of-flight mass spectrometry (UHPLC-QTOF/MS). Results: Principal component analysis (PCA) of the spectra showed clear metabolic changes between the two groups. Thirty six potential biomarkers associated with CTCL were found. Conclusions: Based on PCA, several biomarkers were determined and further identified by LC/MS/MS analysis. All of these could be potential early markers of CTCL. In addition, we established that heparin as anticoagulant has better pre-treatment results than EDTA with the UHPLC-QTOF/MS appraoch.
\end{abstract}

Keywords: Cutaneous T cell lymphoma - metabolomics - biomarkers - UHPLC-QTOF/MS

Asian Pac J Cancer Prev, 15 (13), 5417-5421

\section{Introduction}

Cutaneous T cell lymphoma (CTCL) is the most common primary malignant $\mathrm{T}$ cell lymphoma of the skin, presenting as erythema, plaques, tumors or erythroderma. Incidence is $1 / 10,000$ people per year. Although, the actual incidence of the disease may be underestimated due to different diagnostic criteria (Chuang et al., 1990; Weinstock et al., 1999). The median onset age of CTCL has been reported as 57 years (Kim et al., 2003). But a report from China states the median onset age as being 47.5 years before 2000, and 34 years from 2001-2008, showing the onset of CTCL tends to be in the young ( $\mathrm{Li}$ et al., 2008). Mycosis fungoides is the most frequent type of CTCL, which has an indolent course initiating as erythema, plaques for years and involving lymph nodes or visceral organs as the disease advances. The aggressive type of CTCL has a poor prognosis (Regina et al., 2002). The etiology of CTCL remains poorly understood, and occupational exposure, virus infection and genetic mutation have been proposed as etiological factors. The aberrant expression and function of the transcriptional factors and regulators of signal transduction have been reported in CTCL (Yin et al., 2013). It has been hypothesized that a dysfunctional regulation of small molecules plays a key role in the malignant formation. The occurrence and development of CTCL is a dynamic process regulated by multiple genes, ultimately leading to tumor metabolic changes. The use of metabolomics to study different blood samples from CTCL patients and healthy volunteers, by searching for small and diagnosis-related molecular biomarkers, may offer the opportunity for early diagnosis of CTCL. In this study, we aim to differentiate the expression of metabolic molecules in CTCL and map the potential biomarkers in CTCL plasma.

LC/MS has been widely applied in the analysis of biological sample according to Kunnathur et al. (Kunnathur et al.,2013).In metabolomics, tandem mass spectrometry was often used to detect as many metabolites as possible. In addition, TOF mass analyzer can provide a more accurate molecular weight of metabolites compared to the others. Ultrahigh performance liquid chromatography (UHPLC)-quadrupole time-of-flight mass spectrometry (QTOF/MS) was employed to profile the plasma of patients with CTCL. Differences in metabolomics data from the two groups were characterized using principal components analysis (PCA). Based on pattern recognition results, we aimed to establish a diagnosis model and explore the potential metabolic biomarkers for early diagnosis and staging in gastric cancer.

Sample pretreatment process is a critical factor for the success of metabolomics research studies. Therefore the major steps in sample pretreatment including plasma extraction method, stability of plasma samples at $25^{\circ} \mathrm{C}$ and anticoagulation in sample collection were investigated. 


\section{Materials and Methods}

\section{Patient recruitment}

This prospective study was approved by the Ethics Committee of Ruijin Hospital, Shanghai Jiaotong University. Informed consent was obtained from all participants. CTCL plasma was obtained from 35 patients and matched plasma taken from healthy volunteers. The skin biopsy was examined by histopathological and immunohistochemical stain and $\mathrm{T}$ cell receptor gene rearrangement. The diagnosis was made by two experienced dermatopathologists. A computerized tomography (CT) scan and lymph node ultrasound examination were used to determine the tumor stage. (Xia et al., 2013) All patients were evaluated by the same dermatologist.

\section{Sample collection}

Venous blood was collected from 30 control subjects and 35 CTCL patients without medication. All the samples were then transferred into heparinized tubes and immediately centrifuged at 12,000 rpm for 10 minutes. Fresh plasma was frozen in liquid nitrogen during operation, then stored at $-80^{\circ} \mathrm{C}$ until processing. To a 150 $\mu \mathrm{L}$ aliquot of plasma samples, $450 \mu \mathrm{L}$ of methanol was added for protein precipitation. After centrifugation at $12,000 \mathrm{rpm}$ for 10 minutes, an aliquot of $10 \mu \mathrm{L}$ supernatant was injected for UHPLC/MS analysis.

\section{UHPLC-QTOF/MS analysis}

Plasma samples were analyzed by an Agilent UHPLCQTOF/MS (Agilent Corporation, Santa Clara, CA, USA). Chromatography was performed on a $100 \times 2.1$ mm ACQUITY UPLC ${ }^{\circledR}$ HSS T31.8 $\mu$ m column (Waters Corporation, Milford, MA, USA). The mobile phase, with $0.3 \mathrm{ml} / \mathrm{min}$ flow rate, consisted of solvent $0.1 \%$ formic acid (Sigma-Aldrich, Germany) and acetonitrile containing $0.05 \%$ formic acid. Gradient elution was performed and the program is shown in Table 1.

Mass spectrometric detection was carried out on an Agilent 1260 Infinity series mass spectrometer (Agilent Corporation). The electrospray ionization source was set in positive and negative modes, respectively. Instrument parameters were set as follows: capillary voltage 3.5 $\mathrm{kV}$, gas temperature $350^{\circ} \mathrm{C}$ and desolvation temperature $350^{\circ} \mathrm{C}$. Nitrogen was used as desolvation and cone gas with a flow rate of $10 \mathrm{~L} / \mathrm{min}$, respectively. Full scan mode was employed in the mass range of 100-1000 amu. In the MS/MS experiments, argon was used as collision gas and collision energy was set according to the construction of metabolites. Data were collected in centroid mode. Table 1. The Program of Gradient Elution

\begin{tabular}{lcrrc}
\hline & Time $(\mathrm{min})$ & $\mathrm{A} \%$ & $\mathrm{~B} \%$ & Flow $(\mathrm{ml} / \mathrm{min})$ \\
\hline Positive mode & 0 & 2 & 98 & 0.3 \\
& 2 & 2 & 98 & 0.3 \\
& 12 & 95 & 5 & 0.3 \\
Negative mode & 22 & 95 & 5 & 0.3 \\
& 0 & 2 & 98 & 0.3 \\
& 2 & 2 & 98 & 0.3 \\
& 12 & 95 & 5 & 0.3 \\
\hline
\end{tabular}

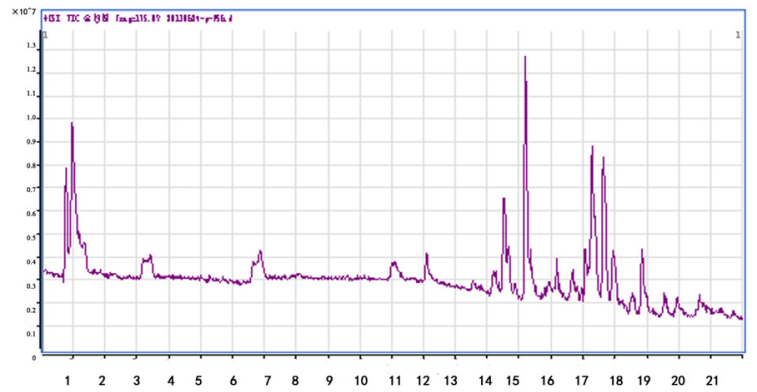

Figure 1. A Typical Ultrahigh Performance Liquid Chromatography-Quadrupole Time-of-Flight Mass Spectrometry (UHPLC-QTOF/MS) Total Ion Chromatogram of Study Sample

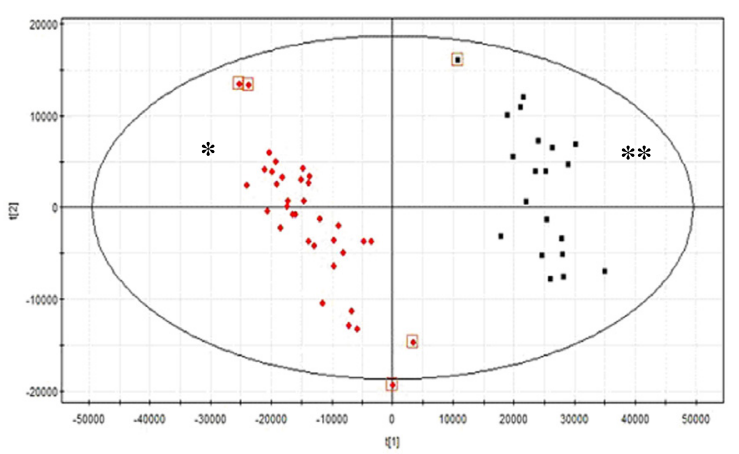

Figure 2. Partial Least Squares-Discriminate Analysis (PLS-DA) of All Data. (*control samples, **study samples)

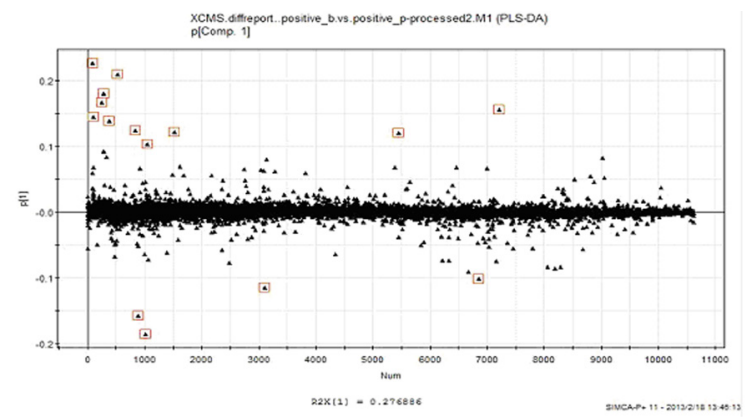

Figure 3. Scatter Plots of All Endogenous Metabolites

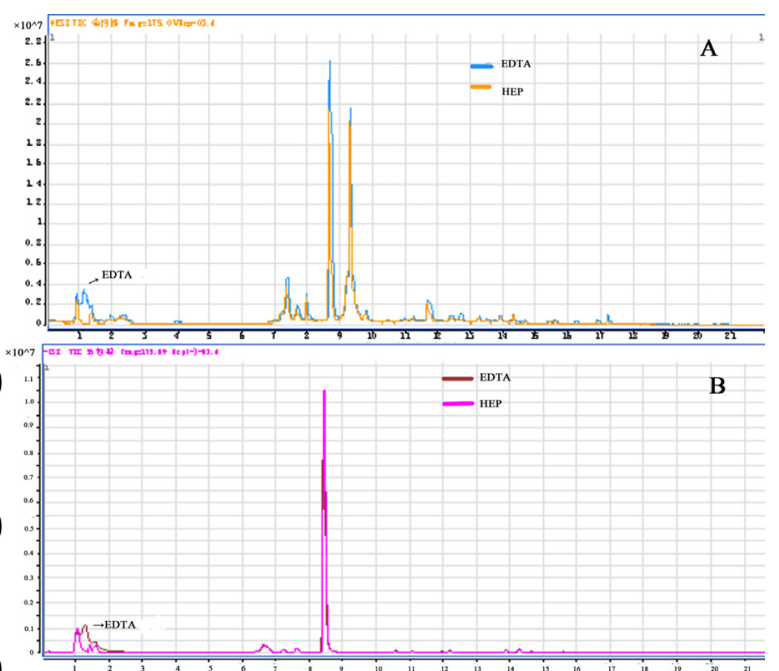

Figure 4. Total Ion Chromatogram of Globulin with EDTA and Heparin in the Positive(A)/Negative(B) mode 
Table 2. Results of comparison between gadobenate dimeglumine with the golden standard $(95 \% \mathrm{CI})$

\begin{tabular}{|c|c|c|c|c|}
\hline \multicolumn{5}{|c|}{ Positive Mode } \\
\hline $\mathrm{M}+\mathrm{H} \mathrm{m} / \mathrm{z}$ & Retention time & Metabolites & MW of metabolites $+\mathrm{H}$ & MW of metabolites \\
\hline 162.12 & 0.93 & Nicotine imine & 162.1151 & 161.1079 \\
\hline 496.35 & 19.34 & LysoPC (16:0) & 496.3398 & 495.3325 \\
\hline \multirow[t]{2}{*}{214.92} & 0.76 & 1,1-Dibromo-2-propanone & 214.8702 & 213.8629 \\
\hline & & Edetic Acid & 293.0979 & 292.0907 \\
\hline \multirow[t]{4}{*}{120.08} & 1.87 & L-Threonine & 120.0655 & 119.0582 \\
\hline & & L-Homoserine & 120.0655 & 119.0582 \\
\hline & & L-Allothreonine & 120.0655 & 119.0582 \\
\hline & & Oxoglutaric acid & 147.0288 & 146.0215 \\
\hline \multirow[t]{3}{*}{175.02} & 0.59 & trans-Aconitic acid & 175.0237 & 174.0164 \\
\hline & & Dehydroascorbic acid & 175.0237 & 174.0164 \\
\hline & & cis-Aconitic acid & 175.0237 & 174.0164 \\
\hline 137.05 & 1.3 & Hypoxanthine & 137.0458 & 136.0385 \\
\hline 243.29 & 15.45 & 1-Hexadecanol & 243.2682 & 242.261 \\
\hline \multicolumn{5}{|c|}{ Negative Mode } \\
\hline $\mathrm{M}-\mathrm{H} \mathrm{m} / \mathrm{z}$ & Retention time & Metabolites & MW of metabolites $+\mathrm{H}$ & MW of metabolites \\
\hline 344 & 1.38 & Guanosine 2',3'-cyclic phosphate & 344.04016 & 345.04743 \\
\hline 297.16 & 22.16 & Minaprine & 297.17209 & 298.17936 \\
\hline \multirow{3}{*}{293.18} & 26.07 & 17-Hydroxylinolenic acid & 293.21222 & 294.2195 \\
\hline & & 13-OxoODE & 293.21222 & 294.2195 \\
\hline & & 9(10)-EpODE & 293.21222 & 294.2195 \\
\hline \multirow[t]{5}{*}{313.05} & 1.25 & Beta-D-Glucopyranuronic acid & 313.05651 & 314.06378 \\
\hline & & 1-Salicylate glucuronide & 313.05651 & 314.06378 \\
\hline & & Geranyl-PP & 313.06115 & 314.06843 \\
\hline & & 5'-Phosphoribosyl-N-formylglycinamide & 313.04424 & 314.05152 \\
\hline & & 7,8-Dihydropteroic acid & 313.10546 & 314.11274 \\
\hline \multirow[t]{3}{*}{300.01} & 1.38 & N-Acetyl-D-galactosamine 1-phosphate & 300.04899 & 301.05627 \\
\hline & & N-Acetyl-D-Glucosamine 6-Phosphate & 300.04899 & 301.05627 \\
\hline & & N-Acetyl-D-mannosamine 6-phosphate & 300.04899 & 301.05627 \\
\hline 315.05 & 1.28 & Olivin & 315.08741 & 316.09469 \\
\hline 298.16 & 22.07 & Hydrocodone & 298.14487 & 299.15214 \\
\hline \multirow[t]{2}{*}{174.96} & 0.89 & Ascorbic acid & 175.02481 & 176.03209 \\
\hline & & D-Glucurono-6,3-lactone & 175.02481 & 176.03209 \\
\hline 565.34 & 21.95 & Nigroxanthin & 565.40511 & 566.41238 \\
\hline 589.34 & 22.06 & D-Urobilinogen & 589.30316 & 590.31044 \\
\hline 554.36 & 23.9 & Pseudoecgonine & 554.3083 & 185.10519 \\
\hline \multirow[t]{2}{*}{409.24} & 25.68 & $\operatorname{LPA}(16: 0 / 0: 0)$ & 409.23606 & 410.24334 \\
\hline & & $\operatorname{LPA}(0: 0 / 16: 0)$ & 409.23606 & 410.24334 \\
\hline 195.81 & 1.2 & Ubiquinone 6 & 195.80389 & 590.43351 \\
\hline
\end{tabular}

\section{Data processing and identification}

LC/MSD ChemStation software (Agilent, Shanghai, China) was used for autoacquisition of LC total ion chromatograms (TICs) (Figure 1) and fragmentation patterns. The accurate mass number of every peak was available by XCMS-Online.

Compounds were mainly identified by searching the Human Metabolome Database (www.hmdb.ca) and by matching $\mathrm{m} / \mathrm{z}$ (accurate mass number). Biomarkers were identified using biochemical databases such as KEGG and METLIN.

\section{Statistical analysis}

Qualitative analysis of MassHunter Acquisition Data was used to analyze the experimental data. Results are presented as means \pm standard deviation (SD). Data that were not normally distributed were logarithmically transformed to obtain normal distribution before analysis. Continuous variables were analyzed by one-way ANOVA with Tukey's test. $P>0.05$ was considered to be statistically significant. A logistic regression analysis was performed to obtain the score of significant difference between CTCL and control samples.

\section{Results and Discussion}

\section{Plasma pretreatment}

Plasma sample collection and handling procedures are critical for successful metabolomics research studies (Yin et al., 2013). Firstly, in the sample collection step, commonly used anticoagulants such as ethylenediaminetetraacetic acid (EDTA) and heparin were investigated. In order to avoid plasma matrix interference, globulin samples were added with EDTA and heparin, respectively, to analyze the matrix effect of anticoagulants. The results showed that in either positive or negative mode, an EDTA sample has an impurity peak at $1 \mathrm{~min}$ compared with heparin. Heparin was therefore chosen as anticoagulant in our plasma sample collection. Protein precipitation by methanol or acetonitrile was tested respectively. 
Table 3. Stability and Precision

\begin{tabular}{|c|c|c|c|c|}
\hline \multicolumn{5}{|c|}{ Stability within day } \\
\hline Peak No. & . $\mathrm{m} / \mathrm{z}$ & Compound & $\begin{array}{l}\text { RSD\%(average) } \\
\text { Retention time }\end{array}$ & $\begin{array}{c}n=5 \\
\text { Peak area }\end{array}$ \\
\hline 1 & 137.0458 & Hypoxanthine & $0.577(1.458)$ & 6.68 \\
\hline 2 & 242.261 & 1-Hexadecanol & $0.2249(11.119)$ & 3.48 \\
\hline 3 & 496.348 & LysoPC(16:0/0:0) & $0.254(14.411)$ & 2.29 \\
\hline 4 & 524.3792 & LysoPC(18:0/0:0) & $0.213(15.635)$ & 8.65 \\
\hline \multicolumn{5}{|c|}{ Precision results } \\
\hline Peak No. & . $\mathrm{m} / \mathrm{z}$ & Compound & RSD\%(average) & $\mathrm{n}=5$ \\
\hline 1 & 137.0458 & Hypoxanthine & $0.440(1.356)$ & 5.33 \\
\hline 2 & 242.261 & 1-Hexadecanol & $0.09(11.190)$ & 5.96 \\
\hline 3 & 496.348 & LysoPC(16:0/0:0) & $0.055(14.332)$ & 3.42 \\
\hline 4 & 524.3792 & LysoPC(18:0/0:0) & $1.570(15.502)$ & 4.22 \\
\hline
\end{tabular}

\section{Optimization of UHPLC/MS method}

Different mobile phase systems were investigated, such as formic acid-methanol and formic acid-acetonitrile. Considering the separation and running time, the mobile phase adopted in our study consisted of solvent $0.1 \%$ formic acid and acetonitrile containing $0.05 \%$ formic acid with gradient elution program.

\section{Precision and stability}

The applied method was validated prior to the analysis of the experimental samples, including the precision of injection, the within-day stability and the repeatability of sample preparation. Extracted ion chromatographic peaks of four ions $(\mathrm{m} / \mathrm{z} 137.0458,1.356 \mathrm{~min} ; \mathrm{m} / \mathrm{z} 242.2610$, $11.190 \mathrm{~min} ; \mathrm{m} / \mathrm{z} 496.3480,14.332 \mathrm{~min} ; \mathrm{m} / \mathrm{z} 524.3792$, $15.502 \mathrm{~min}$ ) distributed in different spectrum regions and retention time were selected for the method validation. Compared with the standards (retention time and $\mathrm{m} / \mathrm{z}$ ), four compounds were identified among the 36 potential biomarkers: Hypoxanthine, 1-Hexadecanol, LysoPC (18:0/0:0) and LysoPC (16:0/0:0). The relative standard deviations (RSDs) of peak intensities and retention time for the selected ions in pooled plasma sample were calculated.

\section{UHPLC/MS metabolite profiles}

The preparation of plasma samples for metabolic profiling analysis by UHPLC/MS involved a protein precipitation step to extract low-molecular weight compounds and remove the large amounts of proteins that would otherwise interfere with the UHPLC/MS analysis. More quantitative information was obtained from the positive ion mode than that collected under the negative ion mode, so that molecular ions $(\mathrm{M}+\mathrm{H})+$ accounted for the majority of the mass spectrum. Figure 1 shows the representative positive base peak intensity.

\section{Analysis of UHPLC/MS data}

In order to find the metabolites with a significant change (i.e., potential biomarkers), partial least squaresdiscriminate analysis (PLS-DA) was constructed using the metabolite intensities as variables. As a classic unsupervised method (no prior knowledge concerning groups or tendencies within the data sets was necessary) for pattern recognition, PLS-DA was expected to pick out distinct variables as potential biomarkers through statistical protocols. The score plot for PLS-DA of control and CTCL patients is shown in Figure 2. Two groups were separated with a clear border. The results showed that the method could find potential biomarkers and distinguish CTCL patients from healthy people.

\section{Biomarker identification}

In the LC/MS TICs of samples from the study and control groups, the majority of the peaks were identified as endogenous metabolites based on the Human Metabolome Database, including amino acids, organic acids, inorganic acids, carbohydrates, fatty acids, aldehydes, amines, amides, polyols and pyrimidines (Wu et al., 2010). There were about 600 signals obtained.

SIMCA- $P$ was used to find metabolites whose contents were significantly different between CTCL and control samples (Figure 3). According to the analysis results, we chose absolute values with $P>0.05$ as potential biomarkers (Table 2).

\section{Study of stability and precision}

Extracts of plasma samples were stored at $25^{\circ} \mathrm{C}$ and injected at $0,4,8,12$ and 24 hours, respectively. The RSDs of peak intensities and retention time for the selected ions in pooled plasma sample were calculated (Table 3) (Yang et al., 2013).

Precision of injection was carried out by the continuous detection of five injections of the same standard sample which was considered a potential biomarker. RSDs ranged from $0.09-1.6 \%$ for retention time and from 3.5-6.0\% for peak intensity. The results are shown in Table 3.

\section{Study of matrix effect}

Globulin samples were added with EDTA and heparin respectively to analyze the matrix effect of anticoagulants (Figures 4) (Yang et al., 2013). Either in positive or negative mode, EDTA sample has an impurity peak at 1 min compared with heparin. Therefore, we chose heparin as the anticoagulant based on its better pre-treatment results.

\section{Acknowledgements}

This work was supported by a grant from the National Science Foundation Project of China (KRF301100).

\section{References}

Chuang TY, Su WP, Muller SA (1990). Incidence of cutaneous $\mathrm{T}$ cell lymphoma and other rare skin cancers in a defined population. J Am Acad Dermatol, 23, 254-6.

Kim YH, Liu HL, Mraz-Gernhard, S et al (2003). Long-term outcome of 525 patients with mycosis fungoides and Sezary syndrome: clinical prognostic factors and risk for disease progression. Arch Dermatol, 139, 857-66.

Kunnathur MS, Chandrasekaran G (2013). Acacia ferruginea inhibits tumor progression by regulating inflammatory mediators-(TNF-a, iNOS, COX-2, IL-1ß, IL-6,IFN- $\gamma$, IL-2, GM-CSF) and pro-angiogenic growth factor-VEGF. Asian Pac J Cancer P, 14, 3909-19

Li Ying, Yuan WR, Shen XY, Zheng J(2008). The comparison of the efficacy of skin directed therapy versus chemotherapy 
for CTCL. J Clin Dermatol, 37, 91-3.

Regina FP, Paulus Z, Barbara B et al (2002). Primary cutaneous lymphomas: applicability of current classification schemes (European Organization for Research and Treatment of Cancer, World Health Organization) based on clinicopathologic features observed in a large group of patients. Blood, 99, 800-5.

Weinstock MA, Gardstein B (1999). Twenty year trends in the reported incidence of mycosis fungoides and associated mortality. Am J Public Health, 89, 1240-4.

Wu H, Xue RY, Tang ZQ et al (2010). Metabolomic investigation of gastric cancer tissue using gas chromatography/mass spectrometry. Anal Bioanal Chem, 396, 1385-95.

Xia YH, Li m, Fu DD et al (2013). Effects of PTTG downregulation on proliferation and metastasis of the SCL-1 cutaneous squamous cell carcinoma cell line. Asian Pac J Cancer $P, \mathbf{1 4}, 6245-48$.

Yang W, Chen YH, Xi C et al (2013). Liquid chromatographytandem mass spectrometry-based plasma metabolomics delineate the effect of metabolites' stability on reliability of potential biomarkers. Anal Chem, 85, 2606-10.

Yin PY, Andreas $P$, Holger F et al (2013). Preanalytical aspects and sample quality assessment in metabolomics studies of human blood. Clin Chem, 59, 5833-845. 